\title{
Effects of ECM Proteins and Cationic Polymers on the Adhesion and Proliferation of Rat Islet Cells
}

\author{
Guoping Chen*, Naoki Kawazoe and Tetsuya Tateishi
}

Biomaterials Center, National Institute for Materials Science, 1-1 Namiki, Tsukuba, Ibaraki 305-0044, Japan

\begin{abstract}
The effects of extracellular matrix (ECM) proteins and cationic polymers on the adhesion and proliferation of rat islet cells, RIN-5F cells, were investigated. ECM proteins of laminin, fibronectin, vitronectin, type I collagen, type II collagen, and type IV collagen, and cationic polyelectrolytes of poly(L-lysine) and poly(allylamine) were coated on the wells of polystyrene cell culture plates. Their effects on the adhesion and proliferation of RIN-5F in serum-free and serum mediums were compared. The cell number on the laminin-coated surface was the highest among the coated surfaces. Laminin promoted cell adhesion more strongly than did the other ECM proteins and cationic polyelectrolytes. Vitronectin, type IV collagen, and poly(L-lysine) showed moderate effects, but type I collagen and type II collagen did not have any effects on adhesion and proliferation compared with the uncoated polystyrene cell culture plate. Fibronectin promoted cell adhesion but not cell proliferation. Cationic poly(allylamine) had an inhibitory effect in serum-free medium and for longterm culture in serum medium. The ECM proteins of laminin, vitronectin, and type IV collagen, and cationic poly(Llysine) will be useful for the surface modification and construction of biomaterials and scaffolds for islet cell culture and tissue engineering.
\end{abstract}

\section{INTRODUCTION}

Diabetes mellitus is one of the most prevalent and destructive diseases in the world, and affects more than 171 million individuals [1]. Islet transplantation and tissue engineering have been developed as alternatives to the more conventional treatments such as insulin administration $[2,5]$. Lack of an adequate supply of islets and favorable biomaterials hinders the progress of these approaches. Development of new culture methods and cell sources such as pancreatic stem cells and differentiation methods have been tried to help solve the problem of an inadequate cell supply [6, 8]. The development of functional biomaterials to promote islet proliferation and facilitate cell adhesion and encapsulation in hydrogels or porous scaffolds is strongly anticipated $[9,11]$.

A strategy to control the function of pancreatic islet cells in vitro is to use the surface chemistry by designing an optimal surface for cell culture in either two or three dimensions $[12,13]$. When biomaterials and scaffolds are exposed to biological environments, extracellular matrix (ECM) proteins are non-specifically adsorbed to the surface of nearly all the biomaterials, then cells indirectly interact with the biomaterial surface through the adsorbed ECM proteins. Surface modification with various ECM proteins such as laminin, fibronectin, vitronectin, collagen, etc. has been used to mimic the in vivo microenvironment to control cell function $[14,15]$. Biomaterials and scaffolds can be coated or chemically bonded with these proteins and molecules.

Besides ECM proteins, the surface properties, especially electrostatic properties, have been reported to have effects on cell functions such as adhesion, proliferation, and differentiation [16-19]. Polyelectrolytes such as cationic and anionic

\footnotetext{
*Address correspondence to this author at the Biomaterials Center, National Institute for Materials Science, 1-1 Namiki, Tsukuba, Ibaraki 305-0044, Japan; E-mail: Guoping.CHEN@nims.go.jp
}

polyelectrolytes have been used to modify the surfaces of biomaterials and scaffolds. Cationic poly(D-lysine) and poly(ethylene-imine) have been introduced on biomaterial surfaces to promote cell adhesion by the electrostatic attraction between the positive charge of the biomaterial surface and negatively charged cells [20].

ECM proteins and cationic polyelectrolytes may also be used as biomaterials and scaffolds for islet cell culture. However, their effects on the adhesion and proliferation of islet cells are not clear. Therefore, in this study, several kinds of ECM proteins and cationic polyelectrolytes were coated on polystyrene cell culture plates and their effects on islet cells studied. RIN-5F cells, a secondary clone of the rat islet tumor cell line RIN-m, were used for cell culture. RIN-5F cells secrete insulin and are a model for the study of the biology of pancreatic islet cells, specifically, the mechanisms controlling the synthesis, storage, and secretion of insulin $[21,22]$.

\section{EXPERIMENTAL}

\section{Materials}

Laminin, fibronectin, vitronectin, polyallylamine (Mw 60,000), and poly(L-lysine) were purchased from SigmaAldrich Inc. (St. Louis, MO, USA). Bovine type I, type II, and type IV collagen acidic solutions were purchased from Koken Co., Ltd. (Tokyo, Japan). WST-1 assay was purchase from Roche Diagnostics (Indianapolis, IN, USA). Rat pancreatic $\beta$-cell line, RIN-5F cells (passages $20-26$ ), was purchased from ATCC (Rockville, MD, USA). RPMI 1640 medium was purchased from Dai-Nippon Corp. (Osaka, Japan). Fetal bovine serum (FBS) was purchased from Gibco-BRL (Grand Island, NY, USA). Deionized water was obtained with a Milli-Q Lab Water Filter System from Millipore Corporation (Billerica, MA, USA). 


\section{COATING OF ECM PROTEINS AND CATIONIC POLYMERS}

Aqueous solutions of laminin, fibronectin, and vitronectin were reconstructed with Milli Q water at a concentration of $100 \mathrm{ug} / \mathrm{mL}, 100 \mathrm{ug} / \mathrm{mL}$, and $50 \mathrm{ug} / \mathrm{mL}$, respectively. Poly (L-lysine) and polyallylamine were dissolved in Milli Q water to prepare their aqueous solutions at a concentration of $100 \mathrm{ug} / \mathrm{mL}$. The type I, type II, and type IV collagen were received as solutions. These were diluted with $0.001 \mathrm{M}$ acetic acid to a concentration of $100 \mathrm{ug} / \mathrm{mL}$. The aqueous solutions of these proteins and cationic polymers were sterilized by filtration with a filter having a pore size of $0.22 \mathrm{um}$ and then added to the wells of 96 -well polystyrene cell culture plates $(20 \mu \mathrm{L} /$ well $)$. The 96 -well plates were stored at $-4^{\circ} \mathrm{C}$ for 12 hours. The laminin-, fibronectin-, and collage-coated plates were washed three times with phosphate-buffer solution free of calcium and magnesium ions. The vitronectin-, poly(L-lysine)-, and polyallylamine-coated plates were washed with Milli Q water three times. The plates were then used for cell culture.

\section{CELL CULTURE, CELL ADHESION, AND PROLIF- ERATION ASSAY}

The RIN-5F cells were cultured in RPMI 1640 medium containing $10 \%$ fetal bovine serum, $100 \mathrm{U} / \mathrm{ml}$ penicillin, and $100 \mu \mathrm{g} / \mathrm{ml}$ streptomycin. The subcultured RIN-5F cells were collected by treatment with trypsin/EDTA solution and washed once with RPMI 1640 serum-free medium. The cells were then suspended at a density of $2.0 \times 10^{5}$ cells $/ \mathrm{mL}$ in RPMI 1640 serum-free medium or RPMI 1640 serum medium. The cell suspension solutions in the RPMI 1640 serum-free and serum mediums were added to each well (100 $\mu \mathrm{L} /$ well) of the laminin-, fibronectin-, vitronectin-, type I collagen-, type II collagen-, type IV collagen-, poly(Llysine)-, and polyallylamine-coated plates and an uncoated polystyrene cell culture plate (control). The cells were cultured in the plates in an incubator under an atmosphere of $5 \% \mathrm{CO}_{2}$ at $37^{\circ} \mathrm{C}$. After culture for 8 hours, their morphology was observed by a phase contrast microscope.

Cell adhesion and proliferation were measured using the WST-1 assay. This is a colorimetric assay for the quantification of cell viability and proliferation that is based on the cleavage of a tetrazolium salt (WST-1) by mitochondrial dehydrogenases in viable cells. Increased enzyme activity leads to an increase in the amount of formazan dye, which is measured with a spectrophotometer. After the cells were cultured in the wells of the coated and control plates in serum-free medium for 4 hours and in serum medium for 30 minutes, and 24 and 48 hours, the culture medium was aspirated and $100 \mu \mathrm{L}$ of RPMI 1640 serum medium was added along with $10 \mu \mathrm{L}$ of Cell Proliferation Reagent WST-1. For all time points, a standard curve was developed by plating $1.0 \times 10^{3}, 2.0 \times 10^{3}, 4.0 \times 10^{3}, 6.0 \times 10^{3}, 8.0 \times 10^{3}, 1.0 \times 10^{4}$, $1.5 \times 10^{4}, 2.0 \times 10^{4}, 3.0 \times 10^{4}$, and $5.0 \times 10^{4}$ cells in $100 \mu \mathrm{L}$ of RPMI 1640 serum medium and $10 \mu \mathrm{L}$ of Cell Proliferation Reagent WST-1. The plates were then incubated for an additional 4 hours at $37^{\circ} \mathrm{C}$. After incubation, the absorbance of the samples against the background control on a microtiter plate reader (Bio-Rad Benchmark Plus ${ }^{\mathrm{TM}}$ Microplate Spectrophotometer) was obtained at a wavelength of $440 \mathrm{~nm}$ with a reference wavelength of $650 \mathrm{~nm}$.

\section{RESULTS}

The RIN-5F cells were cultured on the coated and control surfaces in serum-free medium for 8 hours, and their morphology was observed by a phase-contrast microscope. The cells spread well on the laminin-coated surface and slightly on the fibronectin-coated surface. No obvious spread was observed on the vitronectin-, type I collagen-, type II collagen-, type IV collagen-, poly(L-lysine)-, poly(allylamine)coated and control surfaces (Fig. 1). The cells remained separated on the laminin-, fibronectin-, vitronectin-, poly(Llysine)-, poly(allylamine)-coated and control surfaces, but some of the cells on the type I collagen-, type II collagen-, and type IV collagen-coated surfaces aggregated. When cultured in serum medium for 8 hours, cell spread was observed only on the laminin-coated surface. The cells did not spread on the other surfaces (Fig. 2). The cells on the laminin-, poly(L-lysine)-, and poly(allylamine)-coated surfaces were separate. However, some of the cells aggregated on the fibronectin-, vitronectin-, type I collagen-, type II collagen-, type IV collagen-coated and control surfaces. The spread morphology of the cells on the laminin-coated surface in serum-free and serum mediums was similar. The round morphology of the cells on the poly(L-lysine)- and poly(allylamine)-coated surfaces in serum-free and serum mediums was also similar. Cell aggregation was more evident in serum medium than in serum-free medium.
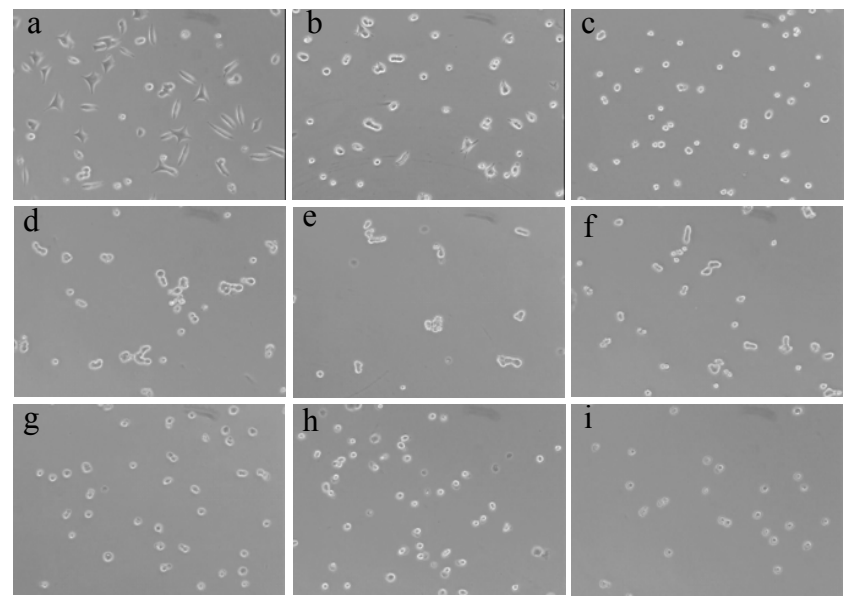

Fig. (1). Phase-contrast micrographs of RIN-5F cells cultured on laminin- (a), fibronectin- (b), vitronectin- (c), type I collagen- (d), type II collagen- (e), type IV collagen- (f), poly(L-lysine)- (g), poly(allylamine)- coated (h), and polystyrene cell culture plate (control) (i) surfaces in serum-free medium for 8 hours.

Cell adhesion on the coated and control surfaces in serum-free medium was measured after 4 hours culture (Fig. 3). The cells adhered to the coated surfaces in the order: laminin- $>$ poly(L-lysine)- $>$ vitronectin- $>$ type IV collagen$>$ fibronectin- $>$ control, $>$ type I collagen- $>$ type II collagen- $>$ poly(allylamine). The laminin-coated surface promoted the adhesion of RIN-5F cells most extensively. The positively charged poly(L-lysine)-coated surface also showed a high promotive effect on cell adhesion. The vitronectin-, type IV collagen-, and fibronectin-coated surfaces showed moderate effects. The type I collagen-coated surface had the same effect as that of the uncoated control surface. The type II collagen-coated surface showed a lower adhesion 
effect than that of the control surface. Almost no cells adhered to the poly(allylamine)-coated surface. The poly(allylamine)-coated surface suppressed the adhesion of the RIN$5 \mathrm{~F}$ cells in serum-free medium.
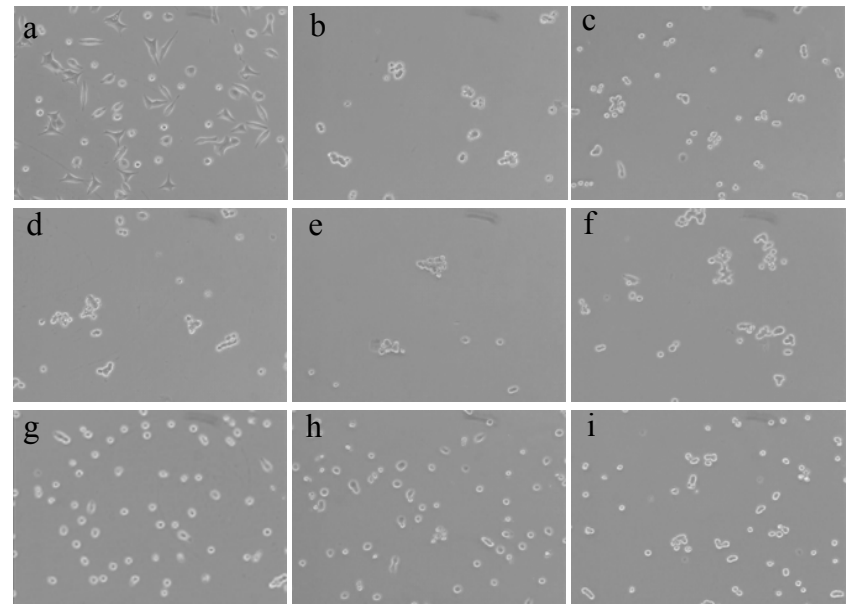

Fig. (2). Phase-contrast micrographs of RIN-5F cells cultured on laminin- (a), fibronectin- (b), vitronectin- (c), type I collagen- (d), type II collagen- (e), type IV collagen-(f), poly(L-lysine)- (g), poly(allylamine)- coated (h), and polystyrene cell culture plate (control) (i) surfaces in serum medium for 8 hours.

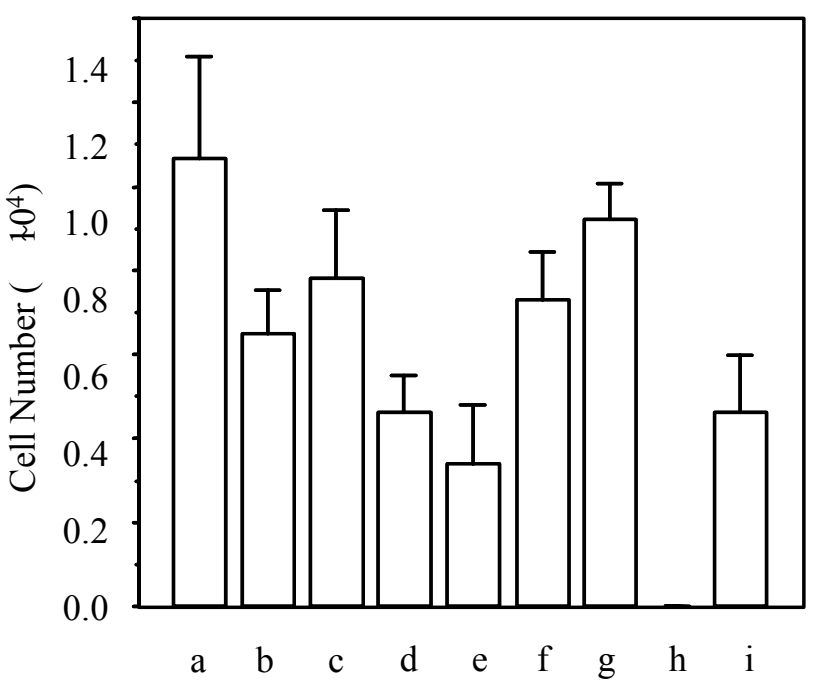

Fig. (3). Adhesion of RIN-5F cells to laminin- (a), fibronectin- (b), vitronectin- (c), type I collagen- (d), type II collagen- (e), type IV collagen- (f), poly(L-lysine)- (g), poly(allylamine)- coated (h), and polystyrene cell culture plate (control) (i) surfaces after culture in serum-free medium for 4 hours. Data represent the average \pm SD of 3 samples.

The adhesion and proliferation of RIN-5F cells on the coated and control surfaces in serum medium were measured after culturing the RIN-5F cells on these surfaces for 30 minutes, 24 hours, and 48 hours (Fig. 4). The cell number on the laminin-coated surface was the highest at all the three time points. The RIN-5F cells adhered quickly and their number increased with culture time on the laminin-coated surface. The poly(L-lysine)- and poly(allylamine)-coated surfaces promoted cell adhesion after culture for 30 minutes in serum medium. The cell number on the poly(L-lysine)- coated surface increased with culture time while cell proliferation on poly(allylamine)-coated surface was suppressed. The type IV collagen-coated surface also promoted cell adhesion and proliferation. Cell adhesion on the vitronectincoated surface was at the same level of those on the type I collagen-, type II collagen-, and control surfaces, but cell proliferation on the vitronectin-coated surface was greater than on those surfaces. Cell adhesion and proliferation on type I collagen-, type II collagen-, and fibronectin-coated surfaces were similar.

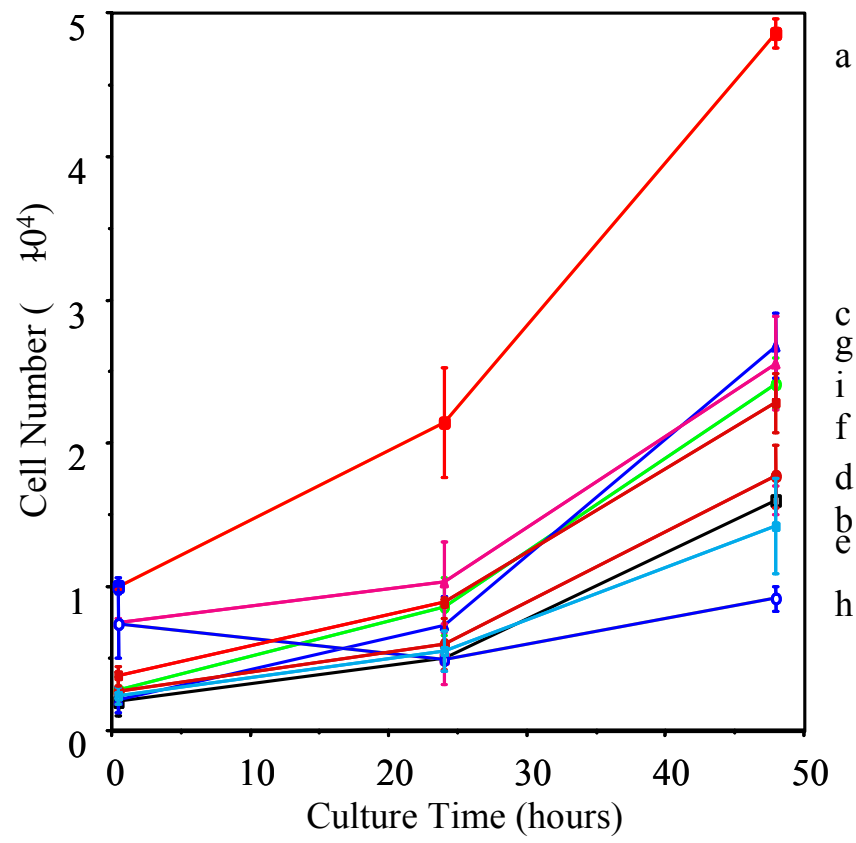

Fig. (4). Cell number of RIN-5F cells on laminin- (a), fibronectin(b), vitronectin- (c), type I collagen- (d), type II collagen- (e), type IV collagen- (f), poly(L-lysine)- (g), poly(allylamine)- coated (h), and polystyrene cell culture plate (control) (i) surfaces in serum medium. Data represent the average \pm SD of 3 samples.

\section{DISCUSSION}

ECM proteins of laminin, fibronectin, vitronectin, type I collagen, type II collagen, type IV collagen, and cationic polyelectrolytes of poly(L-lysine) and poly(allylamine) were coated on the surfaces of polystyrene cell culture plates. Their effects on the adhesion and proliferation of RIN-5F in serum-free and serum mediums were compared. The cell behaviors were dependent on not only the coated surfaces, but also on the culture conditions. The laminin-coated surface promoted cell spread in both serum-free and serum mediums. The fibronectin-coated surface showed some effect on cell spread, but only in serum-free medium. Surfaces coated with other molecules and the control surface did not support cell spread either in the serum-free or serum medium. The cells on laminin-, poly(L-lysine)-, and poly (allylamine)-coated surfaces remained separate in both the serum-free and serum mediums. The cells on the type I collagen-, type II collagen-, and type IV collagen-coated surfaces aggregated in both the serum-free and serum mediums. However, the cells on the fibronectin- and vitronectin-coated surfaces remained separate in serum-free medium, but aggregated in serum. These results indicate the strong interaction between the RIN-5F cells and the laminin- and cationic polymer-coated surfaces. 
The laminin-coated surface had the highest cell number. The type IV collagen-, vitronectin-, and poly(L-lysine)coated surfaces showed moderate effects. The fibronectincoated surface promoted cell adhesion, but cell numer on this surface was low. The type I collagen- and type II collagencoated surfaces showed slight effects on cell adhesion and proliferation in comparison to that of the uncoated control surface. The poly(allylamine)-coated surface promoted cell adhesion in serum medium, but suppressed cell adhesion and cell proliferation in serum-free medium.

Extracellular matrix proteins interact with cells and control cell functions through their binding to integrins, a widely expressed family of cell surface receptors [16, 23]. Laminin, fibronectin, vitronectin, and collagen have been used for surface modification of biomaterials and scaffolds for cell culture of various kinds of cells. Grinnell and Feld demonstrated that fibronectin adsorbed onto tissue culture polystyrene supports greater rates of cell spreading than does bacterial polystyrene [24, 25]. It has also been reported that fibronectin, laminin, and collagen type I interact with the integrins on the surfaces of Schwann cells and support Schwann cell attachment and proliferation [26, 27]. The components of serum may also affect cell function through adsorption to the cell culture substrate or direct interaction with the cells. Serum-free culture might prevent the influence of the serum component. The serum had no influence on a laminin-coated surface, but did have some on other coated surfaces. The cells on other ECM protein-coated surfaces aggregated more easily in serum medium. This might be caused by the adsorption of adhesion-inhibitory factors on these surfaces or the reduction of the number of integrins, as some of the receptors were bound to the serum components. Laminin and type IV collagen are the main components of pancreatic islet basement membrane, which may partially explain the strong effects of these two ECM proteins.

Cationic polymers have been used for surface modification because of their electrostatic interaction with negatively charged cells. The positively charged polyelectrolytes, poly(D-lysine) and poly(ethylene-imine), have been reported to enable attachment of negatively charged Schwann cells [20]. The positively charged poly(L-lysine)- and poly(allylamine)-coated surfaces in this study also showed strong interaction with pancreatic cells. The poly(L-lysine)-coated surface promoted cell adhesion and proliferation both in serum-free and serum mediums. The poly(allylamine)-coated surface did promote cell adhesion in serum medium for a short period. However, its inhibitory effect in serum-free medium and in serum medium for a long period was evident. The cells did not spread on the cationic polymer-coated surfaces. This result coincides with other reports that the attachment of primary rat marrow stromal cells to a positively charged surface in serum medium was enhanced, but their ability to spread was suppressed. These results indicate that while the cationic poly(L-lysine) could promote cell adhesion and proliferation, poly(allylamine) might be toxic to the RIN-5F cells. The effects of ECM proteins and cationic polyelectrolytes on adhesion and proliferation of RIN-5F cells are elucidated in this study. Their effects on insulin secretion of RIN-5F cells will be investigated in next study.

\section{CONCLUSIONS}

Development of functional biomaterials to control the function of islet cells is strongly anticipated for islet transplantation and tissue engineering. ECM proteins of laminin, fibronectin, vitronectin, type I collagen, type II collagen, and type IV collagen, and cationic polyelectrolytes of poly(Llysine) and poly(allylamine) were coated on polystyrene cell culture plates and their effects on rat islet cells, RIN-5F cells, were compared. Laminin showed more promotive effect on the adhesion of RIN-5F cells than did that of other ECM proteins and cationic polyelectrolytes. Cell number was the highest on lamini-coated surface. Vitronectin, type IV collagen, and poly(L-lysine) showed moderate effects, but type I collagen and type II collagen showed no effects in comparison with an uncoated polystyrene cell culture plate. Fibronectin promoted cell adhesion, but not cell proliferation. Cationic poly(allylamine) had some inhibitory effects on RIN-5F cells. The ECM proteins of laminin, vitronectin, type IV collagen, and cationic poly(L-lysine) can be considered candidate biomaterials for islet cell culture and tissue engineering.

\section{ACKNOWLEDGEMENTS}

This work was supported in part by the Ministry of Education, Culture, Sports, Science and Technology of Japan and in part by the New Energy and Industrial Technology Development Organization of Japan.

\section{REFERENCES}

[1] Beck J, Angus R, Madsen B, et al. Islet encapsulation: strategies to enhance islet cell functions. Tissue Eng 2007; 13(3): 589-99.

[2] Efrat S. Cell replacement therapy for type 1 diabetes. Trends Mol Med 2002; 8(7): 334-39.

[3] Cui W, Kim DH, Imamura M, et al. Tissue-engineered pancreatic islets: culturing rat islets in the chitosan sponge. Cell Transplant 2001; 10(4-5): 499-502.

[4] Maria-Engler SS, Mares-Guia M, Correa ML, et al. Microencapsulation and tissue engineering as an alternative treatment of diabetes. Braz J Med Biol Res 2001; 34(6): 691-7.

[5] Hayashi H, Inoue K, Shinohara S, et al. New approach by tissue engineering for extended selective transplantation with a pancreatic B-cell line (MIN6). Transplant Proc 1998; 30(1): 83-5.

[6] Narushima M, Kobayashi N, Okitsu T, et al. A human beta-cell line for transplantation therapy to control type 1 diabetes. Nat Biotechnol 2005; 23(10): 1274-82.

[7] Lock LT, Tzanakakis ES. Stem/Progenitor cell sources of insulinproducing cells for the treatment of diabetes. Tissue Eng 2007; 13(7): 1399-412.

[8] Hess D, Li L, Martin M, et al. Bone marrow-derived stem cells initiate pancreatic regeneration. Nat Biotechnol 2003; 21(7): 763 70.

[9] Dufour JM, Rajotte RV, Zimmerman M, et al. Development of an ectopic site for islet transplantation, using biodegradable scaffolds. Tissue Eng 2005; 11(9-10): 1323-31.

[10] Bloch K, Lozinsky VI, Galaev IY, et al. Functional activity of insulinoma cells (INS-1E) and pancreatic islets cultured in agarose cryogel sponges. J Biomed Mater Res A 2005; 75(4): 802-9.

[11] Dufrane D, Steenberghe M, Goebbels RM, et al. The influence of implantation site on the biocompatibility and survival of alginate encapsulated pig islets in rats. Biomaterials 2006; 27(17): 3201-8.

[12] Sakai S, Ono T, Ijima H, et al. Modification of porous aminopropyl-silicate microcapsule membrane by electrically-bonded external anionic polymers. J Biomater Sci Polym 2003;14(7): 643-52.

[13] Kessler L, Legeay G, West R, et al. Physicochemical and biological studies of corona-treated artificial membranes used for pancreatic islets encapsulation: mechanism of diffusion and interface modification. J Biomed Mater Res 1997; 34(2): 235-45. 
[14] Shin H, Jo S, Mikos AG. Biomimetic materials for tissue engineering. Biomaterials 2003; 24(24): 4353-64.

[15] Morra M. Biochemical modification of titanium surfaces: peptides and ECM proteins. Eur Cell Mater 2006; 12: 1-15.

[16] Keselowsky BG, Collard DM, Garcia AJ. Integrin binding specificity regulates biomaterial surface chemistry effects on cell differentiation. Proc Natl Acad Sci USA 2005; 102(17): 5953-7.

[17] Lan MA, Gersbach CA, Michael KE, et al. Myoblast proliferation and differentiation on fibronectin-coated self assembled monolayers presenting different surface chemistries. Biomaterials 2005; 26(22): 4523-31.

[18] McClary KB, Ugarova T, Grainger DW. Modulating fibroblast adhesion, spreading, and proliferation using self-assembled monolayer films of alkylthiolates on gold. J Biomed Mater Res 2000; 50: 428-39.

[19] Curran JM, Chen R, Hunt JA. The guidance of human mesenchymal stem cell differentiation in vitro by controlled modifications to the cell substrate. Biomaterials 2006; 27(27): 4783-93.

[20] Vleggeert-Lankamp CL, Pêgo AP, Lakke EA, et al. Adhesion and proliferation of human Schwann cells on adhesive coatings. Biomaterials 2004; 25(14): 2741-51.

[21] Nielsen DA, Welsh M, Casadaban MJ, et al. Control of insulin gene expression in pancreatic beta-cells and in an insulin-producing cell line, RIN-5F cells. I. Effects of glucose and cyclic AMP on the transcription of insulin mRNA. J Biol Chem 1985; 260(25): 135859.

[22] Welsh M, Nielsen DA, MacKrell AJ, et al. Control of insulin gene expression in pancreatic beta-cells and in an insulin-producing cell line, RIN-5F cells. II. Regulation of insulin mRNA stability. J Biol Chem 1985; 260(25): 13590-4.

[23] Hynes RO. Integrins: a family of cell surface receptors. Cell 1987; 48(4): 549-54.

[24] Grinnell F, Feld MK. Adsorption characteristics of plasma fibronectin in relationship to biological activity. J Biomed Mater Res 1981; 15(3): 363-81

[25] Grinnell F, Feld MK. Fibronectin adsorption on hydrophilic and hydrophobic surfaces detected by antibody binding and analyzed during cell adhesion in serum-containing medium. J Biol Chem 1982; 257(9): 4888-93.

[26] Baron-Van Evercooren A, Kleinman HK, Seppa HE, et al. Fibronectin promotes rat Schwann cell growth and motility. J Cell Biol 1982; 93(1): 211-6.

[27] McGarvey ML, Baron-Van Evercooren A, Kleinman HK, et al. Synthesis and effects of basement membrane components in cultured rat Schwann cells. Dev Biol 1984; 105(1): 18-28. 